\title{
O ESTADO DA BASE DE DADOS GRAVIMÉTRICOS DO OBSERVATÓRIO NACIONAL (BDG-ON) SITUAÇÃO EM JUNHO, 2001
}

\author{
Walter H. S. Piña ${ }^{1} \&$ Mauro A. de Sousa ${ }^{2}$
}

Received November 26, 2001 / Accepted October 22, 2002

Foi estabelecido no Observatório Nacional, um projeto de desenvolvimento técnico, com a finalidade de estabelecer uma base de dados de gravidade, aberta à comunidade nacional e internacional. O projeto ainda em desenvolvimento visa o registro da informação gravimétrica de campo, a redução dos dados para a remoção de componentes de erro sistemáticas, o ajustamento das observações e finalmente a introdução de todas as informações numa base relacional de dados. Um passo importante do projeto foi concluído em maio 2001, com o estabelecimento dos parâmetros principais da base de dados e o ajustamento das observações pertencentes à Rede Gravimétrica Fundamental do Brasil ao datum absoluto JILAG-3. Na sequiência foi realizado o processamento das observações realizadas com o gravímetro LC\&R G061, no período 1969-1975, totalizando aproximadamente 1500 novas estações. O presente trabalho descreve este processamento mencionado e o estado atual do projeto.

Palavras-chave: Base de dados gravimétricos; Rede Gravimétrica Fundamental do Brasil; Ajustamento; JILAG-3; Gravimetria absoluta.

STATUS OF THE GRAVIMETRIC DATABASE OF THE OBSERVATÓRIO NACIONAL (BDG-ON) - SITUATION AS JUNE, 2001 - A project was set up at the Observatório NacionalON to establish a gravity database open to the national and international communities. This is an ongoing project aiming at recording the raw fieldwork information, performing the conventional data reduction and the adjustment of the gravity intervals. An important by-product of this project was finished in May, 2001 with the adjustment of the Brazilian Fundamental Gravity Network to the absolute datum JILAG-3 and the establishment of the main parameters of the gravimetric database. Next, the observations of the LC\&R G061 gravity meter collected between 1969-1975, nearly 1500 stations were processed and adjusted. This paper reports the adjustment mentioned and the present status of the project.

Key words: Gravimetric database; Fundamental Gravity Network of Brazil; Adjustment; JILAG-3; Absolute gravimetry.

\author{
'OBSERVATÓRIO NACIONAL




\section{INTRODUÇÃO}

Ao longo de várias décadas, o Observatório Nacional tem estado envolvido com o estabelecimento de padrões de gravidade e o seu uso em aplicações metrológicas, geofísicas e geodésicas, no Brasil e em vários países sul-americanos, colaborando com as principais instituições de cada pais. Assim sendo, o ON tem estabelecido bem mais do que 6000 estações de gravidade no Brasil. Na medida que o volume de dados coletados aumentava, foi reconhecida a necessidade de organizá-los em forma mais eficiente, referidos a um único e bem definido datum e integrados numa base de dados que possibilitasse a fácil procura e análise deles, satisfazendo assim as necessidades de pessoas ou instituições públicas e privadas.

O projeto que almeja cumprir estes objetivos começou em agosto 2000, tendo definido os parâmetros principais da base de dados e efetuado o processamento e ajuste dos dados da Rede Gravimétrica Fundamental Brasileira (RGFB) ao datum definido pelo gravimétro absoluto JILAG-3 (Torge et al., 1994). Esta etapa foi concluída em maio 2001 (Subiza Piña \& De Sousa, 2001). Na sequiência, foram processados e incorporados ao ajustamento os dados do gravímetro LC\&R G061, coletados no período 1968-1975 (De Sousa \& Moreira, 1994). Nas seguintes seções são apresentados os resultados do novo ajustamento realizado, incluindo as estatísticas correspondentes.

\section{O AJUSTAMENTO DA REDE GRAVIMÉTRICA}

A RGFB foi ajustada a 9 estações absolutas JILAG-3 e uma relativa (esta última para fechamento de um vínculo internacional), usando o método paramétrico de mínimos quadrados. Esta rede tem um desenvolvimento de uns $39,5^{\circ}$ em latitude, com uma diferencia máxima de aproximadamente 1900 mGal estando conectada à Bolívia, Venezuela e Uruguai (Figura 1).

Após o ajustamento mencionado, as informações do G061 foram processadas. O software GRR.FOR (De Sousa \& Veiga, 2001) foi usado para levar em consideração os efeitos da deriva estática e dinâmica, e formar os intervalos médios de gravidade entre estações. Os efeitos sistemáticos da atração da maré luni-solar foram corrigidos através do Método de Longman (Longman, 1959). O fator de escala usado para o G061 foi o derivado do ajustamento da RGFB. A Figura 2 apresenta a RGFB em conjunto com os dados do G61 adicionados neste ajustamento.

Os intervalos de gravidade escalados foram ajustados por Método de Mínimos Quadrados (MMQ), obtendo-se valores de $g$ e desvios padrão para cada estação. Os residuais padronizados obtidos passaram o teste $\mathrm{c}^{2}$ com nível de confiança de 0,01 (68\%), indicando que uma distribuição normal deles não poderia ser rejeitada.

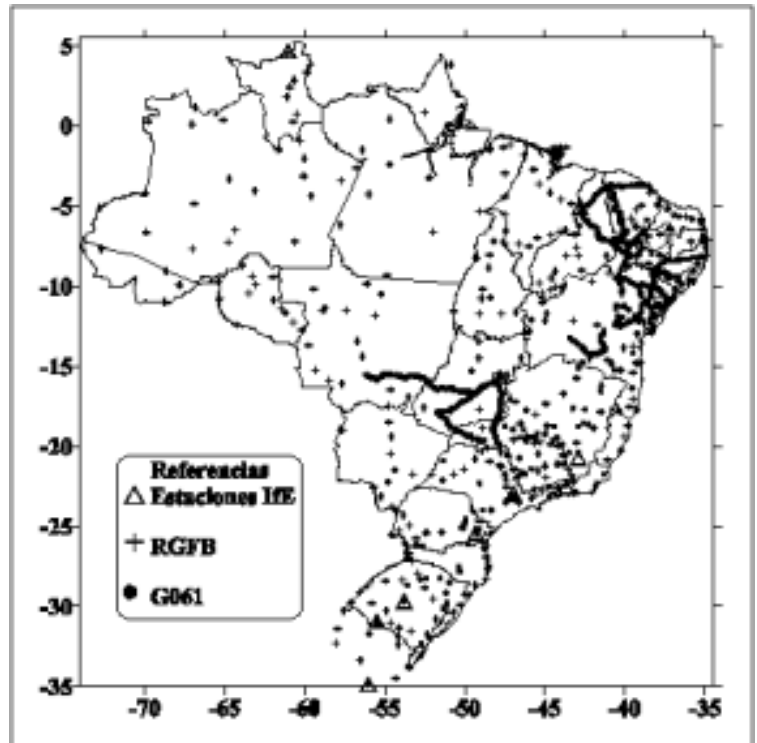

Figura 1 - A RGFB do ON e os dados do acervo G61, adicionados para o novo ajustamento.

A Tab. 1 mostra a totalidade de intervalos de gravidade disponíveis, ajustados e rejeitados, assim como os fatores de escala usados para cada gravímetro.

\begin{tabular}{|c|c|c|c|c|c|}
\hline $\begin{array}{c}\text { No. } \\
\text { LC\&R }\end{array}$ & $\begin{array}{c}\text { Intervalo } \\
\text { Observ. }\end{array}$ & Válido & $\begin{array}{c}\% \\
\text { usado }\end{array}$ & Rejeitado & $\begin{array}{c}\text { Fator } \\
\text { Escala }\end{array}$ \\
\hline G011 & 22 & 15 & 0.4 & 7 & 099995 \\
\hline G013 & 16 & 14 & 0.4 & 2 & 099860 \\
\hline G041 & 51 & 51 & 15 & 0 & 1000761 \\
\hline G061 & 2043 & 2013 & 95.7 & 30 & 100000 \\
\hline G257 & 520 & 513 & 15.4 & 7 & 1000317 \\
\hline$G 454$ & 19 & 19 & 05 & 0 & 1000000 \\
\hline$G 602$ & 296 & 292 & 8.7 & 4 & 1000471 \\
\hline$G 622$ & 250 & 245 & 73 & 4 & 1000448 \\
\hline$G 628$ & 3 & 1 & 003 & 2 & 1000460 \\
\hline$G 674$ & 139 & 135 & 40 & 4 & 1000476 \\
\hline$G 703$ & 16 & 16 & 0.4 & 0 & 100065 \\
\hline Total & 3375 & 3315 & - & 60 & - \\
\hline$\%$ & 100 & - & 98.3 & 1.7 & - \\
\hline
\end{tabular}

Tabela 1 - Estatísticas dos intervalos de gravidade e fatores de escala de cada gravímetro usado no ajustamento. 
A matriz de peso usada no ajustamento foi formada da seguinte forma: i desvios padrão conhecidos das estações absolutas (Torge et al., 1994) e da estação Montevideo (Subiza Piña et al., 1998); ii desvio padrão médio de $20 \mathrm{mGal}$ para os vínculos entre as estações absolutas e a RGFB; iii desvio padrão médio de $25 \mathrm{mGal}$ para os intervalos medidos na RGFB; iv desvio padrão médio de $75 \mathrm{mGal}$ para as observações do G61 adicionadas. Nenhuma estação foi fixada no ajustamento e um conjunto de 10 estações foram injuncionadas em função da inversa do seu desvio padrão (Tab. 2). A variância a posteriori resultante do ajustamento foi de 1,006, confirmando a escolha da matriz de peso adotado.

\begin{tabular}{|c|c|c|c|c|c|}
\hline Estaçăo & Lat. & $\begin{array}{c}\text { Long } \\
\text { (1) }\end{array}$ & $\begin{array}{c}\text { Alitude } \\
\text { (m) }\end{array}$ & $\begin{array}{l}\text { 4 absohuto } \\
\text { (mGal) }\end{array}$ & $\sigma(\mathrm{mGal})$ \\
\hline IfE 040 & +467 & -6107 & 93 & 97722064 & 000027 \\
\hline IfE 112 & -5.08 & -4280 & $\pi$ & 970016343 & 00031 \\
\hline IfE 122 & -1567 & -4783 & 1100 & 970048.798 & 00005 \\
\hline IfE 132 & -20.73 & -4288 & 653 & 978460233 & 000032 \\
\hline IfE 142 & -2255 & -4365 & 400 & 978637583 & 00019 \\
\hline IfE $1 S 2$ & -2298 & -4700 & 850 & 978563771 & 00000 \\
\hline IfE 162 & -25.42 & -4927 & 910 & 978760387 & 00000 \\
\hline IfE 173 & -2968 & -5380 & 85 & 979261635 & 00021 \\
\hline IfE 212 & -3088 & -5553 & 213 & 979344377 & 00014 \\
\hline 250187 & -3485 & -5603 & 16 & 979732398 & 00005 \\
\hline
\end{tabular}

Tabela 2 - Locação, $g$ e desvio padrão das estações absolutas do JILAG-3 e Montevidéu ( $\mathrm{N}^{\text {o. }}$ 250187), usadas no ajustamento da rede de gravidade do $\mathrm{ON}$.

Um resumo das estatísticas dos resultados do ajustamento é apresentado na Tab. 3 .

\begin{tabular}{|c|c|c|c|c|}
\hline Estaçöes absohutas & \multicolumn{4}{|l|}{9} \\
\hline Estaçöes Relativas & \multicolumn{4}{|l|}{1977} \\
\hline Gravímetros absohulos & \multicolumn{4}{|l|}{1} \\
\hline Gravímetmos relativos & \multicolumn{4}{|l|}{11} \\
\hline Estaçöes fixas & \multicolumn{4}{|l|}{0} \\
\hline Fegistro de estaçö̀es & \multicolumn{4}{|l|}{1986} \\
\hline Intervalos de gravidade & \multicolumn{4}{|l|}{3315} \\
\hline Incógnitas & \multicolumn{4}{|l|}{1986} \\
\hline Paxâmetro (jLGaI) & Média & Máx & Mín. & o. \\
\hline Residuais & +051 & 12741 & -180.36 & 20.3 \\
\hline Desvio padrăo das es tacoùes & 150 & 520 & 1 & - \\
\hline
\end{tabular}

Tabela 3 -Estatísticas do ajustamento da RG-ON.

\begin{tabular}{|c|c|c|c|c|}
\hline Gravimeter & Mean & Max & Min & Std. Dev. \\
\hline G011 & +22 & 79.9 & -755 & 48.1 \\
\hline G013 & -19 & 35.6 & -25.7 & 20.0 \\
\hline G041 & -2.4 & 35.3 & -395 & 20.5 \\
\hline G061 & +15 & 127.4 & -180.4 & 19.0 \\
\hline$G 257$ & -02 & 98.9 & -1105 & 20.6 \\
\hline$G 454$ & -19 & 34.8 & -34.7 & 19.3 \\
\hline$G 602$ & -35 & 72.7 & -75.8 & 20.1 \\
\hline$G 622$ & +0.7 & 86.1 & -989 & 23.1 \\
\hline$G 674$ & -25 & 90.0 & -835 & 22.3 \\
\hline G703 & -0.5 & 29.0 & -29.1 & 18.7 \\
\hline Total & +0.5 & 127.4 & -180.4 & 20,3 \\
\hline
\end{tabular}

Tabela 4 - Estatísticas dos residuais (em mGal).
A Tabela 4 mostra os residuais obtidos no ajustamento e por gravímetro, enquanto as Figs. 3 e 4 mostram os histogramas da totalidade dos residuais e especificamente do gravímetro G061.

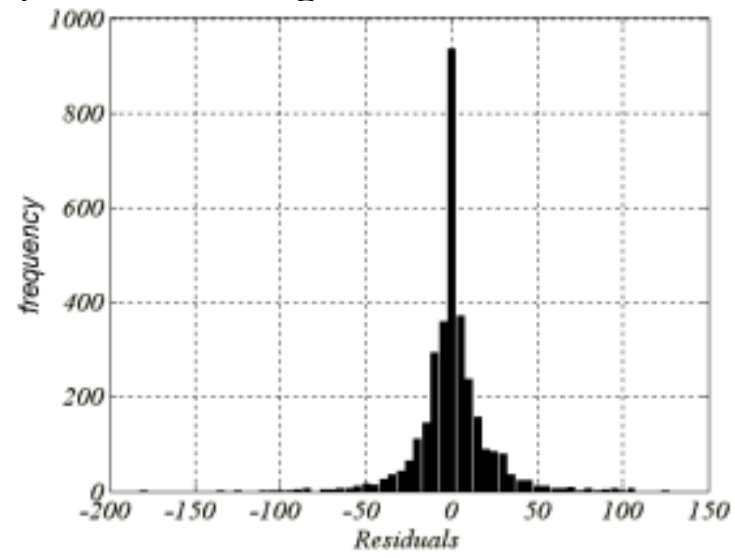

Figura 2 - Histograma dos residuais (3251 dados), em $\mu$ gals.

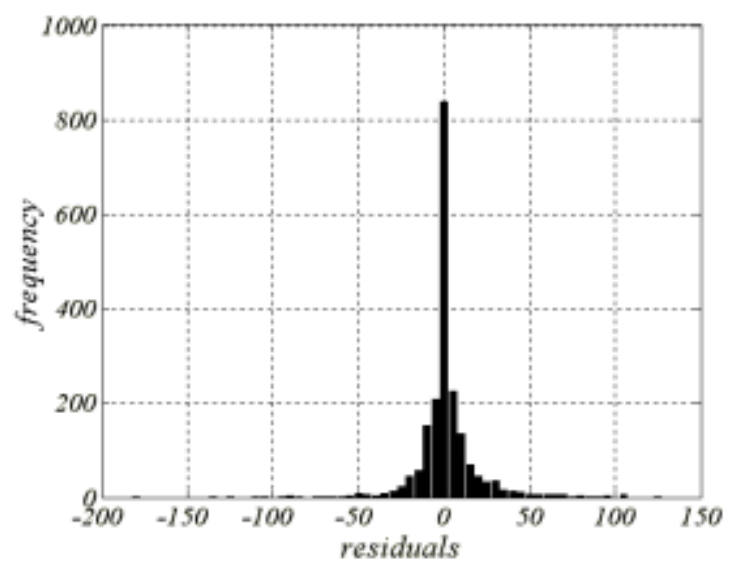

Figura 3 - Residuais do LC\&R G 61 (2013 dados), em $\mu$ gals.

Finalmente na Tab. 5 são apresentados os valores ajustados e incertezas das estações absolutas e na Tab. 6 mostra-se a comparação dos valores obtidos nas estações comuns, pertencentes a rede IGSN71 e a rede gravimétrica do $\mathrm{ON}(\mathrm{RG}-\mathrm{ON})$. As diferenças encontradas entre valores de $g$ ajustados para estas estações são menores ou iguais as próprias incertezas no datum IGSN71, mostrando uma concordância muito boa entre os dois sistemas no Brasil.

\begin{tabular}{|c|c|c|c|c|c|}
\hline Estaçăo & Lat. & $g$ ajustada & $\sigma$ & Residual & Vín. \\
\hline IfE 040 & +467 & 9778220844 & 00029 & -00004 & 3 \\
\hline IfE 112 & -5.08 & 9700163425 & 00003 & +00000 & 2 \\
\hline IfE 122 & -1567 & 9700487979 & 00005 & +00001 & 3 \\
\hline IfE 132 & -20.73 & 9784602331 & 000034 & $-0,0031$ & 10 \\
\hline IfE 142 & -2255 & $9765:$ & 000022 & $-0,0024$ & 14 \\
\hline IfE 152 & -2298 & 97856 & 000032 & +00061 & 14 \\
\hline IfE 162 & -25.42 & 9787603871 & 00002 & -00001 & 27 \\
\hline IfE 173 & -2968 & 9792616349 & 00003 & +00011 & 24 \\
\hline IfE 212 & -3088 & 9793443774 & 00015 & -00004 & 3 \\
\hline 250187 & -3485 & 979732382 & 00102 & $-0,012$ & 5 \\
\hline
\end{tabular}

Tabela 5 - Valor de $g$ ajustado, incerteza e número de vínculos para as estações absolutas e Montevidéu (em mGal). 


\begin{tabular}{|c|c|c|c|c|c|}
\hline $\begin{array}{l}\text { Estaçao } \\
\text { IGSNT I }\end{array}$ & $\begin{array}{c}\text { Lat. } \\
\text { (c) }\end{array}$ & $\begin{array}{l}g \text { ajustado } \\
\text { (mGal) }\end{array}$ & $\begin{array}{l}g \text { IGSNVI } \\
\text { (mGal) }\end{array}$ & $\underset{\text { IGSNT } 1}{\sigma}$ & $\begin{array}{l}\text { Dife- } \\
\text { rença }\end{array}$ \\
\hline $32918 \mathrm{~A}$ & -1.43 & 9700222371 & 97002224 & 0039 & -000 \\
\hline $33039 \mathrm{~J}$ & -3.07 & 970006.145 & 970005.16 & 0052 & -001 \\
\hline $32838 \mathrm{~J}$ & -3.72 & 9700678256 & 97006781 & 0036 & $+0,02$ \\
\hline 3297 & -7.32 & 970031.1700 & 970031.11 & 0053 & +006 \\
\hline $32884 \mathrm{~J}$ & -8.08 & 9781512566 & 97815125 & 0034 & +001 \\
\hline $36428 \mathrm{~B}$ & -1297 & 9763113749 & 97831131 & 0042 & +006 \\
\hline $36479 \mathrm{~J}$ & -1763 & 9785114796 & 978511.46 & 0038 & $+0,02$ \\
\hline $36503 \mathrm{~J}$ & -1990 & 976385558 & 97838550 & 0043 & +0006 \\
\hline $40100 \mathrm{~B}$ & -2030 & 9786418165 & 97864183 & 0042 & -001 \\
\hline $40111 \mathrm{~J}$ & -2168 & 978174698 & 97871749 & 0035 & -0002 \\
\hline $40123 \mathrm{~A}$ & -2288 & 9787898557 & 97878990 & 0027 & $-0,04$ \\
\hline $40136 \mathrm{I}$ & -2338 & 978627276 & 97862729 & 0036 & -001 \\
\hline $40178 \mathrm{~A}$ & -2758 & 979112.4370 & 979123 & 0042 & +005 \\
\hline $43001 \mathrm{~B}$ & -3003 & 9793049614 & 97930500 & 0041 & $-0,04$ \\
\hline $43812 \mathrm{~B}$ & -31.75 & 9794665942 & 97946663 & 0039 & $-0,04$ \\
\hline $43846 \mathrm{~K}$ & -3489 & 979731.4242 & 97973156 & 0043 & -0.14 \\
\hline
\end{tabular}

Tabela 6 - Comparação do datum RG-ON com o datum IGSN71 (em mGal).

\section{CONCLUSÕES}

O projeto de padronização e atualização do acervo gravimétrico, completou mais um passo importante nos seus objetivos, ao incorporar as observações feitas com o gravímetro G061 no período 1969-1975. Um total de 1465 novas estações dispõem de valores de gravidade ajustados no datum absoluto JILAG-3, restando ainda incorporar o acervo observado com o gravímetro Worden. Devido à metodologia de levantamento executado no acervo do G061 e o emprego de apenas um gravímetro, o erro esperado e obtido nas estações novas introduzidas, foi superior ao anterior ajustamento da RGFB. Porém optou-se por aceitá-las levando em consideração o esforço institucional realizado na época e um critério mas realista que simplesmente matemático nos padrões de precisão admitidos. Alguns estudos adicionais devem ser feitos para determinar a possibilidade de recuperar algumas observações e/ou estações que inicialmente foram eliminadas no ajustamento que motiva este relatório, devido a apresentar resíduos ainda de maior magnitude.
A incorporação do desvio padrão dos valores gravimétricos na base de dados, permite oferecer á comunidade de usuários, uma estimativa da confiança nos valores mencionados. O esforço do projeto continuará no futuro no sentido de incorporar os novos dados ajustados, assim como outras complementares destas estações, na Base de Dados Gravimétricos do $\mathrm{ON}$.

\section{REFERÊNCIAS}

De Sousa M.A. \& Veiga, C.H., 2001. Gravity reduction routines for fieldwork data (submitted), Computers \& Geosciences.

De Sousa, M.A. \& Moreira, E.M., 1994. Referring the old gravity network of the National Observatory to the IGSN71/Absolute datum. Part I: The LC\&R 61 data set, Bulletin d'Information Bureau Gravimetrique International, 174: 30-43.

Longman, I.M., 1959. Formulas for computing the tide acceleration due to the Moon and the Sun. J. Geophys. Res. 64: 2351-2356.

Observatório Nacional, 1986. Rede Gravimétrica Fundamental Brasileira 1976-1986. 16 pp.

Torge, W., Timmen, L., Röder, R.H. \& Schmil, M., 1994. The IfE absolute gravity program "South America" 1998-1991. Deustche Geodätische Kommision, B299, Munchen.

Subiza Piña, W.H., Torge, W. \& Timmen, L., 1998. The National Gravimetric Network of Uruguay. Geodesy on the Move. Gravity, Geoid, Geodynamics and Antarctic International Association of Geodesy Symposia, 119 pp.

Subiza Piña, W.H. \& De Sousa, M. A., 2001. The gravity database of the Observatório Nacional and the current status of the Brazilian Fundamental Gravity Network. VXII Congresso Internacional da Sociedade Brasileira de Geofísica, Salvador, Bahia. 